Trauma Berufskrankh 2016 · [Suppl 3]: 18:S226-S230

DOI 10.1007/s10039-015-0074-9

Online publiziert: 21. September 2015

๑) Springer-Verlag Berlin Heidelberg 2015

CrossMark

Christian Lüring

Orthopädische Klinik, Klinikum Dortmund, Dortmund, Deutschland

\title{
Welche Aspekte sprechen für die Knieprothese bei der Gonarthrose?
}

pe der 51- bis 74-Jährigen. Sun et al. [14] beziffern die Prävalenz der Gonarthrose jenseits des 60. Lebensjahres sogar mit etwa $30 \%$. Allerdings weisen lediglich $47 \%$ der Probanden mit radiologischen Zeichen einer Kniearthrose auch die entsprechende klinische Symptomatik auf [14]. Rupprecht et al. [15] konnten zeigen, dass die radiologischen Arthrosezeichen nicht zwingend mit der klinischen Symptomatik und dem intraoperativ festgestellten Schweregrad der Knorpelschädigung korrelierten.

Der Verlauf der Arthrose des Kniegelenks ist in aller Regel fortschreitend. Um die Beschwerden der Betroffenen zu lindern, stehen eine Vielzahl konservativer Behandlungsmöglichkeiten zur Verfügung. Die operativen Möglichkeiten differenzieren sich in gelenkerhaltende und gelenkersetzende Operationen.

Daraus ergibt sich, dass die Behandlung der Arthrose des Kniegelenks facettenreich und auf die individuellen Gegebenheiten des Patienten abgestimmt werden muss. Der Arzt, der die Indikation zur konservativen oder operativen Behandlung und insbesondere zum endoprothetischen Eingriff stellt, benötigt eine Menge an patientenindividuellen Informationen, um festzustellen, wann eine endoprothetische Versorgung medizinisch gerechtfertigt und für den Patienten vertretbar ist.

Wir haben hinsichtlich der Indikationskriterien die deutsche und internationale Literatur gesichtet. Die Auswertung ergab, dass es für den deutschen Sprachraum keine aktuelle, von den Fachgesellschaften konsentierte und einheitlich angewandte Leitlinie gibt. Es steht lediglich eine veraltete S1-Leitlinie zur Verfügung. Die international verfügbare Lite- ratur ist in Hinblick auf ihre Empfehlungen ebenfalls nicht einheitlich.

Zusammenfassend kristallisieren sich anhand der gesichteten Literatur folgende Empfehlungen heraus, die bei der Therapie der Gonarthrose und der Indikationsstellung zum künstlichen Gelenkersatz berücksichtigt werden sollten:

- Die Therapie der Gonarthrose sollte einem multimodalen Ansatz folgen.

- Der Patient muss umfassend über seine Erkrankung informiert und über die Möglichkeiten der Modifikation des Lebensstils aufgeklärt werden.

- Die initiale Therapie beinhaltet eine Kombination aus nichtmedikamentösen und medikamentösen Ansätzen.

- Intraartikuläre Injektionen mit Steroiden sind bei einer akuten Verschlimmerung der Arthrose indiziert.

- Die Indikation zum künstlichen Gelenkersatz besteht, wenn die Schmerzen durch mindestens 6-monatige [16] konservative Maßnahmen nicht ausreichend behandelt werden können, eine erhebliche Einschränkung der Funktion vorhanden ist und typische Arthrosezeichen im konventionellen Röntgenbild vorhanden sind.

Nach Ausschöpfen der konservativen Maßnahmen (Patienteninformation, Ernährungsumstellung, Gewichtsreduktion, Anpassung der Lebensführung, Schmerztherapie, Physiotherapie und ggf. intraartikuläre Injektionen) stellt die Implantation eines künstlichen Kniegelenks mittlerweile eine Standardoperation an vielen Zentren dar. Mit diesem Eingriff können hohe Zufriedenheitsraten erreicht werden. Die Implantation von OberflächenKnie-Totalendoprothesen (TEP) ist mit einer Revisionsrate von unter $5 \%$ inner- 


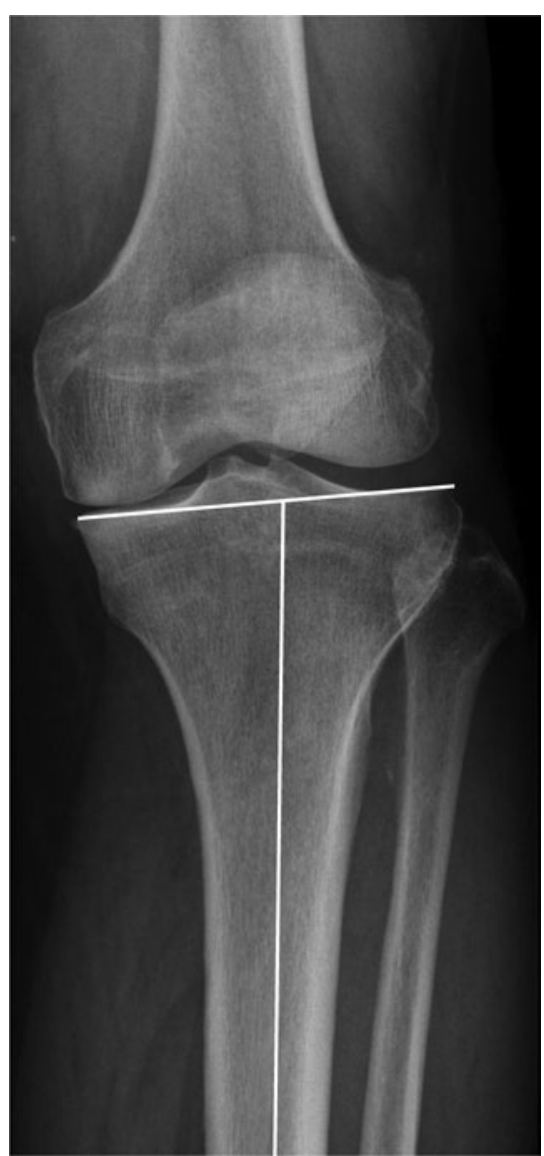

Abb. $1 \Delta$ Mediale Gonarthrose mit pathologisch erniedrigtem proximalem tibialem Gelenkwinkel

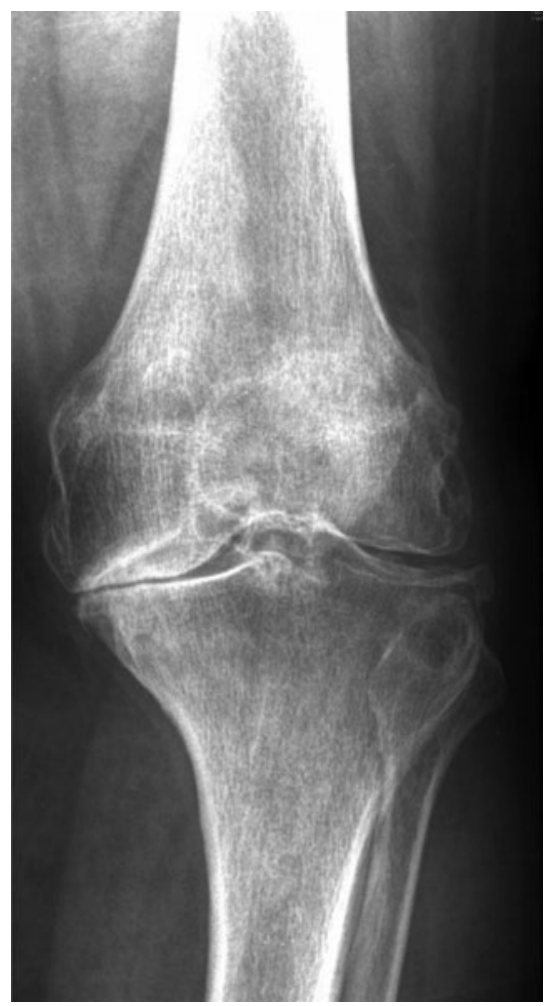

Abb. 4 \ Ubiquitäre Gonarthrose

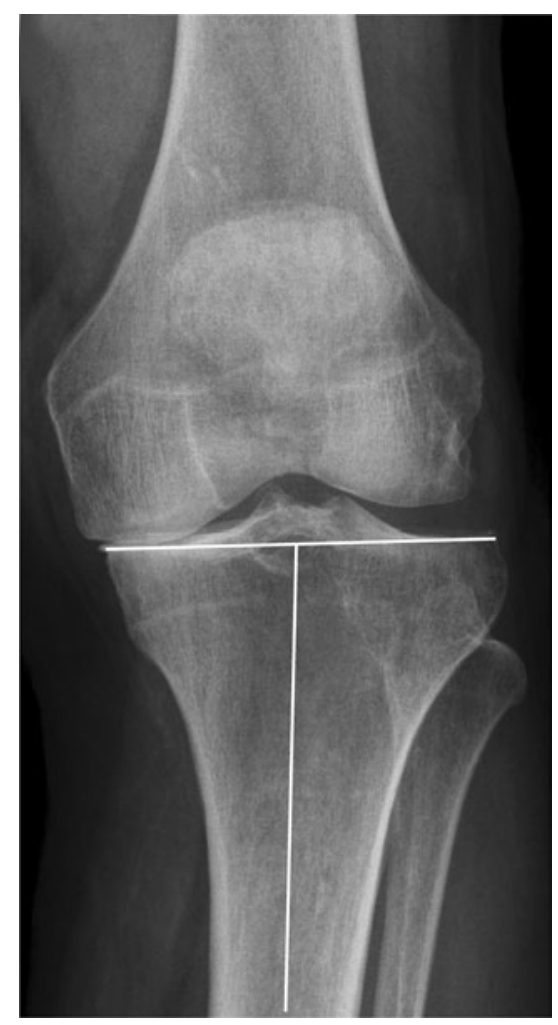

Abb. $2 \Delta$ Mediale Gonarthrose mit normwertigem proximalem tibialem Gelenkwinkel

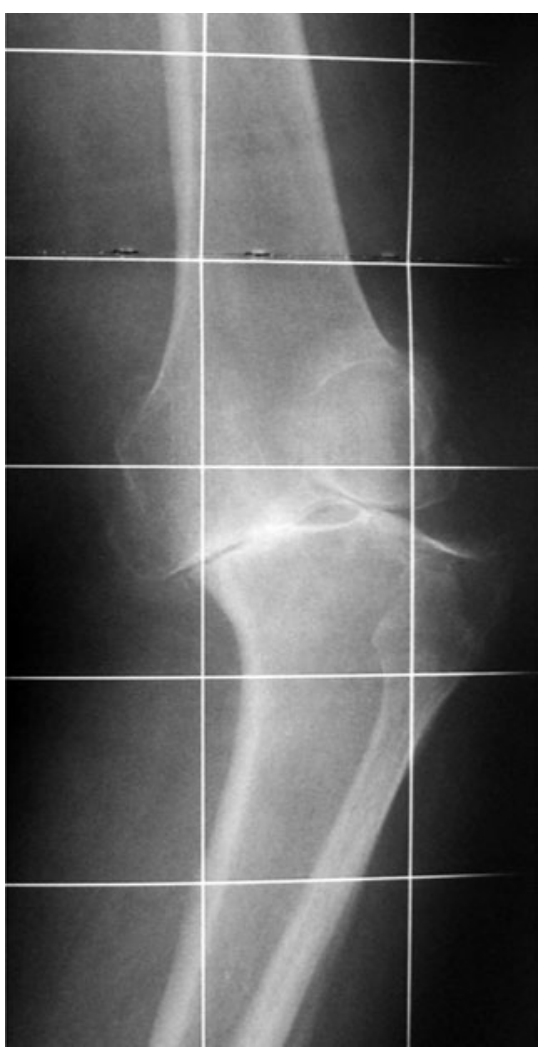

Abb. 5 \ Ubiquitäre Gonarthrose mit erheblicher ligamentärer Instabilität

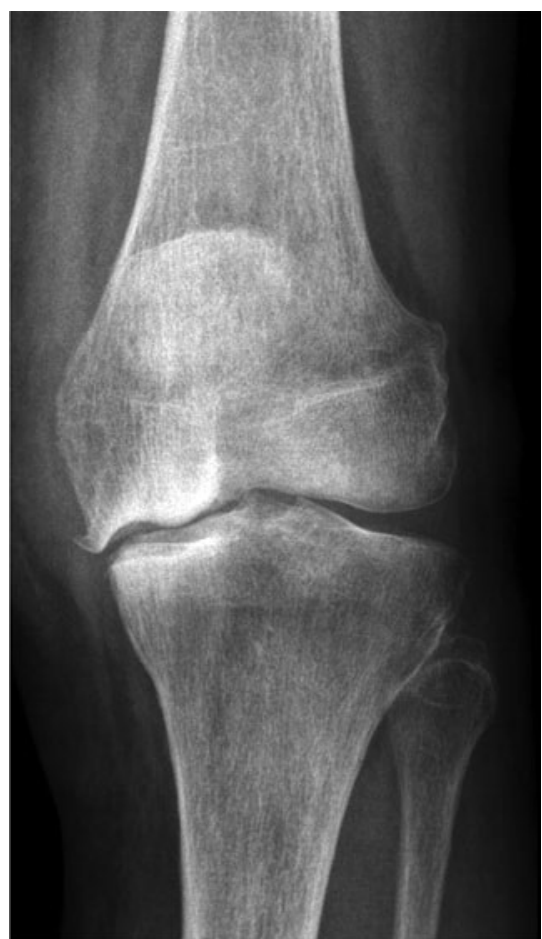

Abb. $3 \triangle$ Mediale Gonarthrose mit Inkongruenz der Gelenkpartner

halb von 10 Jahren und einer Funktionsverbesserung von $30-51 \%$ - je nach verwendetem Score - eine erfolgreiche und dauerhafte Behandlungsmöglichkeit der Gonarthrose [17, 18].

\section{Konservative Therapie der Gonarthrose}

Grundsätzlich wird die Arthrose des Kniegelenks im Sinne einer Stufentherapie behandelt. Im Rahmen der ersten Therapiestufe ist es das primäre Ziel, alle $\mathrm{Pa}$ tienten über die Natur, den Verlauf und die Therapiemöglichkeiten der Erkrankung aufzuklären und ihnen Möglichkeiten der Selbstbehandlung durch Gewichtsreduktion, Bewegungstraining, Anpassung des täglichen Lebens, Schmerzmittel und lokal aufzutragende entzündungshemmende Salben aufzuzeigen. Patienten, die trotz dieser Therapie persistierende Schmerzen haben, können weiterführend mit Physiotherapie, Orthesen, nichtsteroidalen Antirheumatika (NSAR) und anderen Schmerzmitteln behandelt werden. Den wenigen Patienten, die nach Ausschöpfen diese Maßnahmen persistierende Schmerzen haben und entspre- 
chende radiologische Arthrosezeichen aufweisen, kann eine Operation vorgeschlagen werden [19].

\section{Gelenkersatz}

Ist die Arthrose stark fortgeschritten, dann kann ein Gelenkersatz helfen. Die schadhaften Oberflächen im Kniegelenk werden durch eine dem Knie nachempfundene Metalloberfläche (künstliches Kniegelenk) ersetzt. Bei einer streng einseitigen Arthrose kann nur die betroffene Seite (innen/außen) ersetzt werden. Aktuell kann die Standzeit dieser unikondylären Prothesen mit 10-Jahres-Überlebensraten von $84-98 \%$ angegeben werden $[20,21]$. Die Indikation zur Implantation einer Totalendoprothese ist bei bi- oder trikompartimenteller Gelenkdestruktion gegeben, wenn konservative Maßnahmen ausgeschöpft sind und gelenkerhaltende Operationen nicht mehr sinnvoll erscheinen [22, 23]. Zieht man die verfügbaren internationalen Leitlinien und Handlungshinweise zu Rate, dann kristallisiert sich heraus, dass dies dann der Fall ist, wenn die Schmerzen durch mindestens 6-monatige konservative Maßnahmen nicht ausreichend behandelt werden können, eine erhebliche Einschränkung der Funktion vorhanden ist und typische Arthrosezeichen im konventionellen Röntgenbild (Verschmälerung des Gelenkspaltes, knöcherne Anbauten als Ausdruck der Überlastung, vermehrte Sklerosierung der Knochenzone in direkter Nachbarschaft zum Knorpel, Zystenbildung im Knochen) vorhanden sind [18, 24, 25].

Dabei spielen neben den objektivierbaren Kriterien wie dem Ausmaß der Arthrose auf dem konventionellen Röntgenbild, der Stabilität und dem Bewegungsausmaß des Kniegelenks auch die individuellen Beschwerden und der Leidensdruck des Patienten eine Rolle. Wenn objektivierbare Kriterien vorliegen und der Patient nur noch eine schmerzfreie Gehstrecke von wenigen hundert Metern aufweist, regelmäßig Schmerzmittel einnimmt und Belastungs-, Nacht- und Ruheschmerzen angibt, dann sollte die Indikation zum Gelenkersatz gestellt werden [24-27]. Da Schmerzen subjektiv empfunden werden und nur mit einer visuel-

Trauma Berufskrankh 2016 · [Suppl 3]: 18:S226-S230 DOI 10.1007/s10039-015-0074-9

(c) Springer-Verlag Berlin Heidelberg 2015

\section{Lüring}

\section{Welche Aspekte sprechen für die Knieprothese bei der Gonarthrose?}

\section{Zusammenfassung}

Die Arthrose des Kniegelenks ist eine weit verbreitete degenerative Erkrankung, die in aller Regel progredient fortschreitet. Etwa $10 \%$ der Weltbevölkerung ab 60 Jahren leiden an einer symptomatischen Gonarthrose. Die Therapieoptionen sind vielfältig. Zunächst sollten konservative Maßnahmen wie Physiotherapie, Schmerzmedikation, gelenknahe Injektionen und Gewichtsreduktion versucht werden. Sollte ein Nachtschmerz, Ruheschmerz oder initialer Belastungsschmerz bestehen, das Röntgenbild eine fortgeschrittene Arthrose zeigen und die Untersuchung die Befunde bestätigen, ist die Indikation für einen künstlichen Gelenkersatz gegeben. Je nach Ausprägung der Arthrose können Unischlittenprothesen, bikondyläre Prothesen oder gekoppelte Prothesen Verwendung finden.

Schlüsselwörter

Degenerative Erkrankung - Konservative Therapie · Künstliches Kniegelenk · Arthrose · Prothesen

\section{Which aspects indicate total knee replacement for gonarthritis?}

\begin{abstract}
Arthritis of the knee joint is a widely spread degenerative disease, which as a rule progressively advances. The World Health Organization estimated that approximately $10 \%$ of the world population over the age of 60 years suffer from symptomatic arthritis of the knee joint. The therapy options range from conservative treatment (e.g. physiotherapy, analgesics, weight reduction and joint injections) to surgery, such as total joint replacement. Total joint replacement is recommended if the patient suffers from nocturnal pain, pain at rest, immediately after weight bear-
\end{abstract}

ing, if radiological signs of gonarthritis are visible and the clinical examination shows reduction of range of motion and instability of the ligaments. Depending of the degree of arthritis different types of protheses are available: unicompartimental, bicompartimental and if ligaments are unstable, constrained implants.

\section{Keywords}

Degenerative disease - Conservative treatment . Total knee replacement . Arthritis . Prosthesis len Analogskala eingeordnet werden können, ist bislang kein objektivierbarer Konsens vorhanden, wie stark die Schmerzen sein müssen [28]. Die Indikation wird in der Regel individuell gestellt, und auch das Alter, das Körpergewicht und Nebenerkrankungen spielen eine Rolle, da diese Parameter das postoperative Ergebnis beeinflussen [29-32]. Auch die sozialen Bedingungen und häuslichen Versorgungsmöglichkeiten sollen einbezogen werden. Eindeutige und allgemeingültige Indikationskriterien für die Knieprothesenimplantation gibt es nicht [33]. Übereinstimmend geben die European League Against Rheumatism (EULAR) und das amerikanische National Institute of Health (NIH) an, dass bei Dauerschmerzen, die gegenüber medikamentöser Therapie resistent sind, oder auch bei erheblichen Funktionsbeeinträchtigun- gen in Verbindung mit entsprechendem radiologischem Nachweis einer Arthrose eine ausreichende Operationsindikation gegeben ist $[22,23]$. Das genaue Beschwerdeniveau für eine Operationsindikation wird jedoch nicht spezifiziert. Aktuell gibt es keine aktuelle, von den Fachgesellschaften konsentierte gültige deutsche Leitlinie, die die Therapie der Gonarthrose genauer beschreibt.

\section{Radiologische Kriterien}

In Abgrenzung zur hohen Tibiakopfumstellungsosteotomie (HTO) bzw. der distalen femoralen Umstellungsoperation gibt es anatomisch-radiologische Kriterien, die für einen künstlichen Gelenkersatz sprechen. Diese sollen anhand der folgenden Röntgenbilder illustriert werden. Bei einer beginnenden medialen Gon- 
arthrose mit verkleinertem proximalem tibialem Gelenkwinkel bietet sich eher die Umstellungsosteotomie an, da über dieses Verfahren der proximale tibiale Gelenkwinkel normalisiert und die Gelenklinie korrigiert werden kann (• Abb. 1). Demgegenüber sollte bei einer medialen Gonarthrose mit normalem proximalem tibialem Gelenkwinkel eher über eine mediale Unischlittenprothese nachgedacht werden, da eine HTO den proximalen tibialen Gelenkwinkel pathologisch erhöhen würde und damit die Gelenklinie ebenfalls aus der Norm bringen würde. Hier bietet sich also eher das resezierende Verfahren an (• Abb. 2). Eine mediale Gonarthrose mit Inkongruenz der Gelenkanteile sollte ebenfalls mit einem Gelenkersatz - je nach Begleitpathologie mit einer uni- oder bikondylären Oberflächenersatzprothese - versorgt werden (- Abb. 3). Findet sich radiologischerseits eine Arthrose in allen Gelenkanteilen medial, lateral und auch retropatellar -, dann fällt die Entscheidung relativ leicht. Sofern die entsprechenden Kriterien (patientenimmanente Faktoren, ausgeschöpfte konservative Maßnahmen etc.) darauf hindeuten, sollte der Befund mit einer bikondylären Oberflächenersatzprothese versorgt werden (• Abb. 4).

Findet sich eine ubiquitäre Gonarthrose mit zusätzlich erheblicher Bandinstabilität, dann sollte über ein je nach Grad der Instabilität gekoppeltes Implantat nachgedacht werden (• Abb. 5).

\section{Fazit für die Praxis}

- Die Therapie der Gonarthrose folgt zunächst einem multimodalen Ansatz. Es stehen verschiedene, gut untersuchte konservative Therapieformen zur Verfügung. Eine endoprothetische Versorgung ist dann angeraten, wenn

- die radiologischen Kriterien einer fortgeschrittenen Arthrose erfüllt sind,

- der Patient über Nacht-, Ruhe- und einen initialen Belastungsschmerz klagt,

- die Gehstrecke auf wenige $100 \mathrm{~m}$ reduziert ist,

- regelmäßig eine Schmerzmedikation eingenommen wird,
- verschiedene konservative Maßnahmen über zumindest 6 Monate frustran geblieben sind.

- Die endoprothetische Versorgung und die Wahl des Implantates sollten anhand der radiologischen Veränderungen, der Untersuchung des $\mathrm{Pa}$ tienten und der Bedürfnisse des $\mathrm{Pa}$ tienten gewählt werden.

\section{Korrespondenzadresse}

Prof. Dr. med. C. Lüring

Orthopädische Klinik

Klinikum Dortmund

Beurhausstr. 40, 44137 Dortmund

christian.luering@klinikumdo.de

http://www.klinikumdo.de

\section{Einhaltung ethischer Richtlinien}

Interessenkonflikt. C. Lüring gibt an, dass kein Interessenkonflikt besteht.

Dieser Beitrag beinhaltet keine Studien an Menschen oder Tieren.

The supplement containing this article ist not sponsored by industry.

\section{Literatur}

1. Symmons D, Mathers C, Pfleger B (2006) Global burden of osteoarthritis in the year 2000. http:// www.who.int/healthinfo/statistics/bod_osteoarthritis.pdf. Zugegriffen: 03 . März 2013

2. WHO (2003) The burden of musculoskeletal conditions at the start of the new millennium: report of a WHO scientific group. http://www.boneandjointdecade.de/downloads/who_burden_musculoskeletalconditions.pdf. Zugegriffen: 03. März 2013

3. WHO (2013) Chronic diseases and health promotion. Chronic rheumatic conditions. http://www. who.int/chp/topics/rheumatic/en/. Zugegriffen: 02. Mai 2013

4. OECD (2012) Health at a glance: Europe 2012. OECD Publishing. http://dx.doi. org/10.1787/9789264183896-en. Zugegriffen: 02. Mai 2013

5. Buckwalter JA, Lappin DR (2000) The disproportionate impact of chronic arthralgia and arthritis among women. Clin Orthop Relat Res 2000(372):159-168

6. Mannoni A, Briganti MP, Di Bari M, Ferrucci L, Costanzo S, Serni U, Masotti G, Marchionni N (2003) Epidemiological profile of symptomatic osteoarthritis in older adults: a population based study in Dicomano, Italy. Ann Rheum Dis 62(6):576-578

7. Praemer AP, Furner S, Rice DP (1999) Musculoskeletal conditions in the United States. American Academy of Orthopaedic Surgeons, Rosemont

8. Felson D (1995) The epidemiology of osteoarthritis: prevalence and risk factors. In: Kuettner KE, Goldberg VM (Hrsg) Osteoarthritic disorders. American Academy of Orthopaedic Surgeons, Rosemont
9. Dennison E, Cooper CL (2003) Osteoarthritis: epidemiology and classification. In: Hochberg MC, Silman AJ, Smolen JS, Weinblatt ME, Weisman MH (Hrsg) Rheumatology. Toronto, Mosby

10. Lopez AD, Murray CJ et al (2012) Years lived with disability (YLDs) for 1160 sequelae of 289 diseases and injuries 1990-2010: a systematic analysis for the Global Burden of Disease Study 2010. Lancet 380(9859):2163-2196

11. Günther K, Puhl W, Brenner H, Sturmer T (2002) Clinical epidemiology of hip and knee joint arthroses: an overview of the results of the "Ulm Osteoarthrosis Study". Z Rheumatol 61(3):244-249

12. Felson DT (2004) An update on the pathogenesis and epidemiology of osteoarthritis. Radiol Clin North Am 42(1):1-9

13. Hannan MT, Felson DT, Pincus T (2000) Analysis of the discordance between radiographic changes and knee pain in osteoarthritis of the knee. J Rheumatol 27(6):1513-1517

14. Sun Y, Sturmer T, Gunther KP, Brenner H (1997) Incidence and prevalence of cox- and gonarthrosis in the general population. Z Orthop Ihre Grenzgeb 135(3):184-192

15. Rupprecht TN, Oczipka F, Luring C, Pennekamp PH, Grifka J (2007) Is there a correlation between the clinical, radiological and intrasurgical findings of osteoarthritis of the knee? A Prospective study on 103 patients. Z Orthop und Unfall 145(4):430-435

16. Van Manen MD, Nace J, Mont MA (2012) Management of primary knee osteoarthritis and indications for total knee arthroplasty for general practitioners. J Am Osteopath Assoc 112(11):709-715

17. Robertsson $O$, Knutson $K$, Lewold S, Lidgren L (2001) The Swedish Knee Arthroplasty Register 1975-1997: an update with special emphasis on 41,223 knees operated on in 1988-1997. Acta Orthop Scand 72(5):503-513

18. Lutzner J, Kirschner S, Gunther KP, Harman MK (2012) Patients with no functional improvement after total knee arthroplasty show different kinematics. Int Orthop 36(9):1841-1847

19. Lohmander LS, Roos EM (2007) Comment. Clinical update: treating osteoarthritis. Lancet 370:20822084

20. Agneskirchner JD, Lobenhoffer P (2003) Knee joint prosthesis. Chirurg 74(2):M56

21. Deshmukh RV, Scott RD (2001) Unicompartmental knee arthroplasty: long-term results. Clin Orthop Relat Res (392):272-278

22. N.A. (1995) NIH consensus conference: total hip replacement. NIH Consensus Development Panel on Total Hip Replacement. JAMA 273(24):1950-1956

23. N.A. (2004) NIH Consensus Statement on total knee replacement December 8-10, 2003. J Bone Joint Surg Am 86-A(6):1328-1335

24. British Orthopaedic Association, British Association for Surgery of the Knee (1999) Knee replacement: a guide to good practice. http://www.boa. ac.uk/Publications/Documents/tkr_good_practice.pdf. Zugegriffen: 02. Mai 2013

25. Canale S, Beaty J (2008) Campbell's operative orthopaedics, 11. Ausgabe. Mosby Elsevier, Philadelphia

26. Della Valle C, Rosenberg A (2003) Indications for total knee replacement. In: Callaghan J, Rosenberg A, Rubash H, Simonian P, Wickiewicz T (Hrsg) The adult knee. Lippincott Williams \& Wilkins, Philadelphia

27. NIH Consensus Panel (2004) NIH Consensus Statement on total knee replacement December 8-10, 2003. J Bone Joint Surg Am 86-A(6):1328-1335 
28. Lützner J, Kasten P, Günther KP, Kirschner S (2009) Surgical options for patients with osteoarthritis of the knee. Nat Rev Rheumatol 5(6):309-316

29. Amin AK, Clayton RA, Patton JT, Gaston M, Cook RE, Brenkel IJ (2006) Total knee replacement in morbidly obese patients. Results of a prospective, matched study. J Bone Joint Surg Br 88(10):13211326

30. Gillespie GN, Porteous AJ (2007) Obesity and knee arthroplasty. Knee 14(2):81-86

31. Mancuso CA, Ranawat CS, Esdaile JM, Johanson NA, Charlson ME (1996) Indications for total hip and total knee arthroplasties. Results of orthopaedic surveys. J Arthroplasty 11(1):34-46

32. Price AJ, Longino D, Rees J, Rout R, Pandit $H$, Javaid K, Arden N, Cooper C, Carr AJ, Dodd CA, Murray DW, Beard DJ (2010) Are pain and function better measures of outcome than revision rates after TKR in the younger patient? Knee 17(3):196-199

33. Altman RD, Abadie E, Avouac B, Bouvenot G, Branco J, Bruyere O, Calvo G, Devogelaer JP, Dreiser RL, Herrero-Beaumont G, Kahan A, Kreutz G, Laslop A, Lemmel EM, Menkes CJ, Pavelka K, Van De Putte L, Vanhaelst L, Reginster JY (2005) Total joint replacement of hip or knee as an outcome measure for structure modifying trials in osteoarthritis. Osteoarthritis Cartilage 13(1):13-19

\section{Call for Papers: Herbert-Lauter- bach-Preis 2016}

\section{BG Kliniken veranstalten 15.000-Euro-} Wissenschaftswettbewerb

Mit dem Herbert-Lauterbach-Preis würdigen die BG Kliniken auch 2016 wieder herausragende wissenschaftliche Leistungen auf den Gebieten Unfallmedizin und Berufskrankheiten. Im Fokus stehen dabei Themen, die für die medizinische Versorgung von Versicherten der Berufsgenossenschaften und Unfallkassen eine Rolle spielen, wie z.B. typische Verletzungen bei Arbeitsunfällen.

Bewerberinnen und Bewerber um den mit 15.000 Euro dotierten Preis sollten ihre Studien- oder Forschungsarbeiten im Kalenderjahr 2015 entweder in einer Fachpublikation veröffentlicht oder als Manuskript niedergelegt haben. $\mathrm{Zu}$ den erforderlichen Bewerbungsunterlagen gehören vier Druckexemplare der Arbeit in deutscher Sprache, eine kurze Zusammenfassung sowie eine eidesstattliche Erklärung, dass die Arbeit nicht bereits anderweitig ausgezeichnet oder zeitgleich einem weiteren Prüfungsgremium vorgelegt wurde. Die Unterlagen können jedoch auch per E-Mail an HYPERLINK "mailto:forschung@bg-kliniken. de" forschung@bg-kliniken.de oder über das Onlinebewerbungsformular unter HYPERLINK "http://www.bg-kliniken.de/ akutmedizin-rehamedizin/forschung/ herbert-lauterbach-preis/" www.bg-kliniken. de/akutmedizin-rehamedizin/forschung/ herbert-lauterbach-preis/ eingereicht werden.

\section{Bewerbungsschluss ist der 30 . April}

2016.

Bewerbungen per Post senden Sie bitte an:

BG Kliniken - Klinikverbund der gesetzlichen Unfallversicherung gGmbH Stichwort "Herbert-Lauterbach-Preis" Mittelstraße 51

10117 Berlin 